\title{
Nanostructured Antigen-Responsive Hydrogels Based on Peptides for Leishmaniasis Detection
}

\author{
Sivoney F. Souza, ${ }^{a}$ Sergio Kogikoski Jr., ${ }^{a}$ Emerson R. Silva ${ }^{b}$ and Wendel A. Alves*,a \\ ${ }^{a}$ Centro de Ciências Naturais e Humanas, Universidade Federal do ABC, \\ 09210-580 Santo André-SP, Brazil
}

${ }^{b}$ Departamento de Biofísica, Universidade Federal de São Paulo, 04023-062 São Paulo-SP, Brazil

\begin{abstract}
Hydrogels based on peptide nanostructures are biological entities that can be applied in a wide range of applications, such as scaffolds for tissue engineering, drug delivery, and biosensors. The aim of this research was to study peptide hydrogels based on $N$-(9-fluorenylmethoxycarbonyl)$L, L$-diphenylalanine (Fmoc-FF) in two different media: water and phosphate buffer. These hydrogels were used for encapsulating Leishmania infantun chagasi soluble proteins. The structure of the matrices was investigated in detail through scanning and transmission electron microscopy, and small angle X-ray scattering (SAXS). The mechanical behavior of the hydrogels were assessed through rheology assays, demonstrating both the physical and chemical stability of the hydrogel scaffolds. The immunogenicity of immobilized antigens was studied using enzyme-linked immunosorbent assay (ELISA) detection after the reaction with positive and negative dog sera for Leishmania infantum chagasi. The hydrogel was efficient to encapsulate antigens, and can promote the development of novel devices that requires the storage of biomolecules under moist environmental conditions.
\end{abstract}

Keywords: peptide hydrogels, diphenylalanine, immunosensor, ELISA, Leishmaniasis

\section{Introduction}

The development of new biomaterials is a popular subject of research because devices and systems based on these assemblies exhibit strong potential for applications in diagnosis and therapeutics. ${ }^{1-3}$ In particular, hydrogels are a class of materials that have a wide variety of biomedical applications, including biosensing, tissue engineering, regenerative medicine, and drug delivery. ${ }^{4}$ Hydrogel-based structures are expected to have broad potential applications because they are dynamic and responsive systems that play an active role in detecting and delivering architectures and are able to respond to different external stimuli, thereby enabling possibilities for creating so-called smart materials. ${ }^{5-7}$ Among the several possible research topics, the immobilization of biomolecules within hydrogel matrices is a field of intense research activity because the intrinsically hydrated structure of these materials can improve the stability of biomolecules, such as antigens, proteins, or enzymes. In addition, in some cases (depending of the application), hydrogel matrices allow the reuse of the relevant matrix in an environmentally

*e-mail: wendel.alves@ufabc.edu.br friendly manner. ${ }^{8}$ Therefore, these hydrogel matrices open up an exciting new class of biological immobilization materials under hydrated conditions that are designed to maximize the activity of most biological systems.

Among the compounds that self-assemble into ordered hydrogels, those based on short peptides have many interesting properties.9-11 The self-assembly of peptides into hydrogels are usually the result of simple interaction factors, such as the presence of hydrophobic interactions, hydrogen bond donation and reception, and electrostatic interactions. The amino acid structures can overcome all those interactions, and based on design rules, they can be properly synthesized and structured to exhibit different chemical functionalities. ${ }^{1,12}$ The development of the peptide supramolecular structures started at the end of the 1990s with the study of the self-organization of amyloid disease fibers. ${ }^{13-15}$ The self-assembly of the peptides was achieved because of the continual advances in the structural determination of proteins and protein-complexes at the molecular scale; such structural information was then used to design peptides that mimic proteins. ${ }^{16}$

The self-assembly of the peptides into hydrogels usually follow simple steps: after choosing the amino acids 
and synthesizing the expected peptide, it is subsequently self-assembled in solution through specific interactions forming aggregates with specific secondary structures, similar to those of proteins. Furthermore, under specific conditions of concentration, temperature, $\mathrm{pH}$, ionic strength, and others, the secondary structures self-assemble into unitary nanofibers; through intermolecular interactions between these unitary nanofibers, the elongation of the nanofibers occurs to form a hydrogel network, as shown in Figure $1 .{ }^{17}$ Each of these steps can be studied by a variety of spectroscopic, scattering, and microscopic techniques, contributing to the understanding of the formation of the hydrogel and the interactions that govern the self-assembly of peptides. ${ }^{18}$

Because of the diversity of possible structures, many peptides have the relevant structural properties that are required to form stable hydrogels, such as the presence of hydrophobic moieties, charge complementarity, ${ }^{17}$ disulfide bonds, or large aromatic groups, which enhance the gelification capabilities of peptides..$^{10,19,20}$ One approach to achieve such structures involves the introduction of an Fmoc group at the $N$-terminal side of the peptide chain. ${ }^{21,22}$ The Fmoc group is considered a key to the self-assembly peptides into a hydrogel structure, where the peptide chain plays a secondary role in the stabilization of the structure. The Fmoc group drives the stabilization of the structure through strong $\pi$-stacking interactions, which are essential to the formation of the hydrogel. ${ }^{23}$

Among the short peptides, the dimer $L, L$-diphenylalanine (FF) is one of the most studied compounds because of its well-established ability to self-assemble into micronanotubes with hexagonal molecular packing that enables many physical chemical properties. ${ }^{2,24}$ Moreover, it has been demonstrated that the addition of the protecting
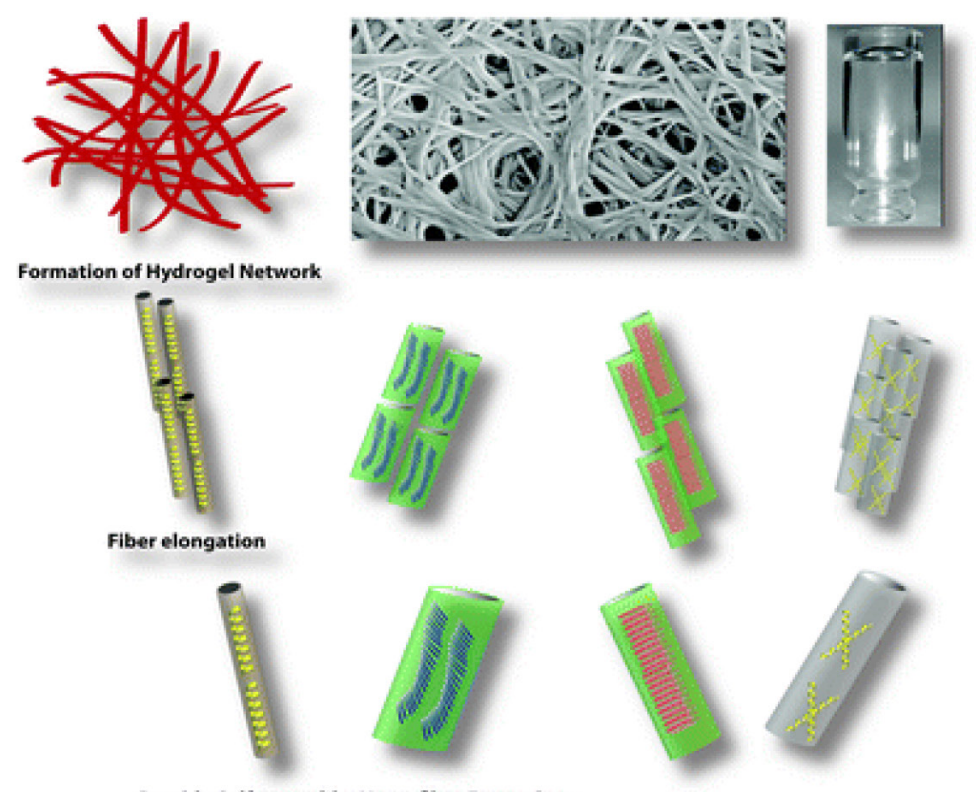

Peptide Self-assembly: Nano-fiber Formation
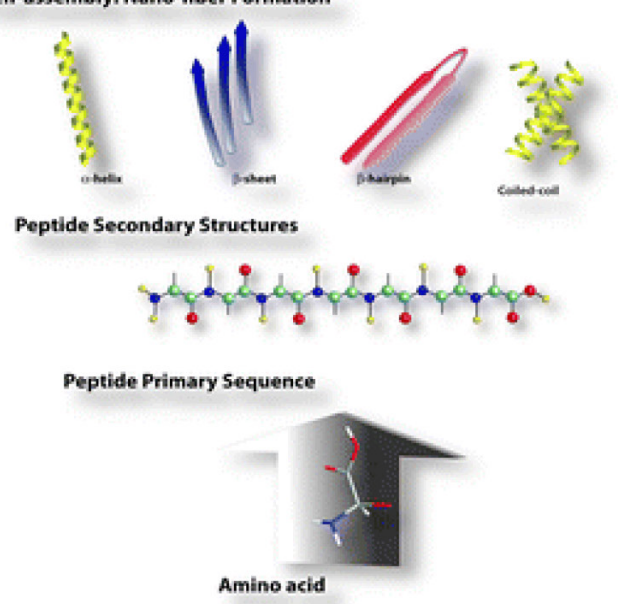

Figure 1. Simplified scheme showing the self-assembly process involved in the formation of peptide-based hydrogels. Reproduced with permission from RSC Advances (Copyright 2016). ${ }^{17}$ 
Fmoc group to the $N$-terminal of the peptide backbone enables the formation of transparent, microporous and very hard hydrogels matrices..$^{25}$ More recently, our group has focused on the study of the physical-chemical properties of FF and the development of novel applications of FF self-assembled materials in many areas, including mechanically reinforced nanocomposites ${ }^{26}$ photocatalyst matrices, ${ }^{27,28}$ biodegradable organic electronic materials, ${ }^{29-31}$ and, principally, electrochemical sensing and biosensing matrices. ${ }^{32-36}$

Fmoc-FF hydrogels offer many advantages for developing sensing devices, the principal one being that biomolecules are immobilized in a hydrated environment without losing its native structure or activity. ${ }^{37,38}$ Thus, using Fmoc-FF hydrogels as encapsulating agents represents an opportunity to design diagnostic sensors to recognize specific diseases with high sensitivity and selectivity. In the literature, the use of Fmoc-FF modified hydrogels for the optical sensing of glucose ${ }^{39}$ and for the electrochemical sensing of hydrogen peroxide released from HeLa cells has already been proposed. ${ }^{5}$ Herein, we demonstrate for the first time the use of an antigen responsive Fmoc-FF hydrogel sensor for neglected Leishmaniasis detection. Many diseases arise in tropical countries; one such disease is Leishmaniasis, which is a widespread zoonotic disease that represents a serious public health issue with various clinical manifestations. According to a disease-burden estimation, Leishmaniasis is the second-ranking cause of mortality and the fourthranking in morbidity among neglected tropical diseases. ${ }^{40}$ As such, immunosensors have attracted great interest in recent years for monitoring biological and chemical agents in a variety of applications. ${ }^{41-43}$

Fmoc-FF has been used to encapsulate and support cells, with a particular focus on biological activity. Herein, Fmoc-FF was designed as a hydrogel for use in the encapsulation of $L$. $i$. chagasi soluble proteins. Encapsulation provides enhanced stability of the antigens, enzymes and other biomolecules because the environmental conditions from the hydrogel persist for long periods, thereby favoring the longer lifetime of this biological system. Therefore, the objective of this work was to produce hydrogels able of encapsulating soluble antigens extracted from promastigotes of $L$. i. chagasi. In addition, it was studied by scanning electron microscopy (SEM), rheology, small- and wide-angle X-ray scattering, transmission electron microscopy (TEM). The antigenicity property of encapsulated proteins was evaluated using enzyme-linked immunosorbent assay (ELISA) through a reaction with specific antibodies from positive and negative dog sera for L. i. chagasi.

\section{Experimental}

\section{Preparation of the hydrogels}

To obtain the Fmoc-FF hydrogel, a similar procedure as that described previously, ${ }^{44}$ but with different solvents, was followed. Hydrogels were prepared by the dissolution of $5 \mathrm{mg}$ of $N$-(9-fluorenylmethoxycarbonyl)$L, L$-dyphenylalanine (Fmoc-FF) in $50 \mu \mathrm{L}$ of 1,1,1,3,3,3-hexafluoro-2-propanol. After the total dissolution of the peptide in the organic solvent, an aqueous solution was added. Next, $950 \mu \mathrm{L}$ of deionized water, PBS, and antigen solution in specific concentrations was added to obtain the pure hydrogel (HO), the hydrogels (HPBS), and the encapsulated antigens in the hydrogel matrix (HA), respectively. All the hydrogels had the same final concentration of peptide of $5 \mathrm{mg} \mathrm{mL}^{-1}$.

\section{Extraction of soluble antigens of Leishmania infantum chagasi}

Leishmania infantum chagasi promastigotes were kept cryopreserved in the Pathogens Laboratory at Universidade Federal do ABC (UFABC). The strains were maintained frozen in liquid $\mathrm{N}_{2}$ and then cultivated at $28{ }^{\circ} \mathrm{C}$ in liver infusion tryptose (LIT) medium (containing fetal bovine serum, antibiotics and $2 \%$ human male urine). Protein extract of L. i. chagasi promastigotes was produced using a similar procedure as that applied by Souto et al..$^{45}$ Promastigotes were harvested in LIT medium. The parasites were washed three times by centrifugation in PBS at $\mathrm{pH} 7.2$ for $10 \mathrm{~min}$. The disruption of the promastigote to release the antigens occurred in three cycles of 5 minutes each at a $40 \mathrm{~W}$ ultrasound treatment in an ice bath. The sonicated culture material was centrifuged at $37000 \times \mathrm{g}$ for $1 \mathrm{~h}$ and 30 min at $4{ }^{\circ} \mathrm{C}$ in an ultra-centrifuge. The supernatant was dialyzed in PBS for $24 \mathrm{~h}$ at $4{ }^{\circ} \mathrm{C}$. The remaining material was filtered in sterile $0.22 \mu \mathrm{m}$ filters under aseptic conditions. The concentration of soluble proteins was measured by the Bradford method.

The Bradford reagent was prepared following the procedure: Coomassie Brilliant Blue G-250 (100 mg) was dissolved in $50 \mathrm{~mL}$ of $95 \%$ ethanol. Next, $100 \mathrm{~mL}$ of $85 \%$ $(\mathrm{m} / \mathrm{v})$ phosphoric acid was added to this solution. The resulting solution was diluted to a final volume of one liter. The final concentrations in the reagent were $0.01 \%(\mathrm{~m} / \mathrm{v})$ Coomassie Brilliant Blue G-250, 4.7\% (m/v) ethanol, and $8.5 \%(\mathrm{~m} / \mathrm{v})$ phosphoric acid.

The calibration curve was obtained with $100 \mu \mathrm{L}$ of bovine serum albumin solutions of known concentrations of $2.0,4.0,6.0,8.0$, and $10 \mu \mathrm{g} \mathrm{mL}^{-1}$ in triplicate with the 
addition of $100 \mu \mathrm{L}$ of Bradford reagent, and then left for 5 minutes to react in environmental conditions.

The intensity of absorbance was obtained using a UV-Vis Varian 50Bio at $595 \mathrm{~nm}$, and the result equation [antigen] $=0.657+0.0439 x$. The antigen concentrations were determined in the materials as 0.55 and $0.38 \mu \mathrm{g} \mathrm{mL}^{-1}$.

\section{Canine serum and infection status}

The canine serum positive and negative samples for L. $i$. chagasi were donated by Dr Arlei Marcili from the Preventive Medicine Department of Medical Veterinary School, Universidade de São Paulo, and were obtained from dogs of visceral Leishmaniasis endemic regions described elsewhere.

\section{Characterization techniques}

\section{Small-angle X-ray scattering (SAXS)}

SAXS data were collected using either the SAXS1 beamline at LNLS, Brazilian Synchrotron Light Laboratory (CNPEM, Campinas, Brazil), or a XEUSS-Xenocs machine at the Institute of Physics of the University of São Paulo (São Paulo, Brazil). At LNLS, we characterized antigen solutions that were loaded into liquid cell sample holders and sandwiched in-between mica windows. Data were obtained at a photon energy of $8 \mathrm{keV}(\lambda=0.154 \mathrm{~nm})$, and data collection was performed using a Pilatus $300 \mathrm{k}$ detector, with a pixel size of $172 \times 172 \mu \mathrm{m}^{2}$. The sampleto-detector distance was $1.2 \mathrm{~m}$, covering a $q$-range from 0.12 to $4.5 \mathrm{~nm}^{-1}$. Twenty frames of 30 seconds each were recorded; if no radiation damage was noticed, then the frames were averaged, and the background was subtracted from the data. Hydrogel samples were loaded into $1.5-\mathrm{mm}$ quartz capillaries and analyzed using the XEUSS-Xenocs instrument operating at photon energy of $8 \mathrm{keV}$. Detection was performed using a Pilatus 300k detector, and the sample-to-detector distance was set at $0.77 \mathrm{~m}$, leading to a $q$-range from 0.11 to $4 \mathrm{~nm}^{-1}$. Four frames of 30 minutes each were recorded and averaged before background subtraction. Data analyses were conducted using model fitting performed with form factor expressions available on the SASFit program. ${ }^{46,47}$

\section{Transmission electron microscopy (TEM)}

The morphology of the self-assembled hydrogel was further assessed by TEM, with the instrument operating at $100 \mathrm{keV}$. To prepare the samples, carbon-coated copper grids (No. 400) were glow discharged for $5 \mathrm{~s}$. The loaded grids were stained with $4 \%(\mathrm{~m} / \mathrm{v})$ uranyl acetate for $60 \mathrm{~s}$ and then analyzed immediately using a transmission electron microscope, JEOL 2100 at LNNano, Brazilian Nanotechnology National Laboratory (CNPEM, Campinas, Brazil).

\section{Scanning electron microscopy (SEM)}

For SEM, the hydrogel samples were placed upon polished metal stubs covered with carbon tape and then dried at environmental conditions. Next, the samples were sputter-coated with Au for 60 seconds in $40 \mathrm{~mA}$. The image acquisition was performed using a scanning electron microscope, FEI Quanta 650 FEG at LNNano, Brazilian Nanotechnology National Laboratory (CNPEM, Campinas, Brazil). The images were analyzed using the ImageJ program to measure fiber diameters and obtain the diameter histogram distribution.

\section{Rheological studies}

Continuous flow and viscoelastic properties of hydrogels were analyzed on a Kinexus rotation rheometer equipped with parallel plate geometry ( $35 \mathrm{~mm}$ diameter) and a sample gap of $0.010 \mathrm{~mm}$ at the temperature of $25^{\circ} \mathrm{C}$, in triplicate. Samples of the hydrogel were carefully applied to the lower plate, ensuring that sample shearing was minimized, and then allowed to equilibrate for $3 \mathrm{~min}$ prior to analysis.

Oscillatory analyses were started by the conduction of a stress sweep, to determine the viscoelastic region of the sample. The analysis was conducted at a constant shear stress of $1 \mathrm{~Pa}$ at frequencies in the range of 0.1 to $10 \mathrm{~Hz}$, which was within the previously determined linear viscoelastic region for all samples, and the storage $\left(G^{\prime}\right)$ and loss (G") moduli were then recorded. The variation of the storage modulus $\left(G^{\prime}\right)$ at low frequencies in a plot of $\mathrm{G}$ " versus $\omega$ follows the power law equation 1 , given by:

$\mathrm{G}^{\prime}=\mathrm{S} \cdot \omega^{\mathrm{n}}$

where $\mathrm{G}^{\prime}$ is the storage modulus; $\mathrm{S}$ the gel strength; $\omega$ the oscillation frequency; and $\mathrm{n}$ is the viscoelastic exponent.

Immunogenicity test of proteins encapsulated in the hydrogels by ELISA

To investigate the immunogenicity of the proteins immobilized or encapsulated by the peptide hydrogel antibodies was conducted via ELISA detection, as described below.

Hydrogels were used with the same quantity of approximately $50 \mu \mathrm{L}$ in each Eppendorf. Six samples of hydrogels with the concentration of $0.344 \mu \mathrm{g} \mathrm{mL}^{-1}$ antigens (HA) and six samples of hydrogels without antigens (HO) 
were used. Three samples of HA and three other samples of $\mathrm{HO}$ hydrogels were incubated with the quantity of $50 \mu \mathrm{L}$ of negative serum with the concentrations of 1:1, 1:10, and 1:40 (v/v) of serum/PBS; the hydrogels that received this specific concentrations of serum were denoted HO1, $\mathrm{HO}$, and HO3, respectively. Another three samples of HA hydrogels and $\mathrm{HO}$ hydrogels were incubated with positive serum in the same concentrations $(1: 1,1: 10$, and 1:40) and were identified as HA1, HA2 and HA3, respectively. These serum solutions were prepared using phosphate buffer solution at $\mathrm{pH}$ 7.4. All the samples were kept in contact overnight.

Next, all the 12 samples were washed 5 times with PBS and then subjected to centrifugation to separate and remove the PBS present. Subsequently, all the samples received the rabbit anti- $\operatorname{dog} \operatorname{IgG}$ for 2 hours and then washed 5 times with PBS. The samples then received the mouse anti- rabbit IgG linked to phosphatase alkaline overnight and then washed 5 times with PBS. Antibody reaction was determined by absorbance intensity at $405 \mathrm{~nm}$ in UV-Vis Varian 50Bio after addition of a substrate for alkaline phosphatase, PNPP (kit from Invitrogen). Figure 2 shows a scheme with the steps of the immunosensing assay.

\section{Results and Discussion}

The peptide Fmoc-FF is characterized by the existence of an aromatic ring in its side chain that allows the establishment of large $\pi$-stacking interactions during the aggregation process. In addition, Fmoc-FF has strong hydrophobic character that facilitates self-organization in aqueous media, being capable of self-assembly into $\beta$-sheet-based hydrogels. ${ }^{48}$ The hydrogels from Fmoc-FF are composed of nanofibers, as observed in SEM images
(Figure 3). Figures 3a and 3b show the hydrogel obtained with deionized water; a homogenous morphology of nanofibers with diameter of approximately $15 \pm 5 \mathrm{~nm}$ is observed. Adding antigens in the hydrogel, as shown in Figures $3 \mathrm{c}$ and $3 \mathrm{~d}$, the structure is quite similar with diameter of approximately $34 \pm 3 \mathrm{~nm}$; based on SEM imaging, it was not possible to observe large differences. Moreover, in Figures 3e and 3f, the SEM images from hydrogel formed in PBS reveal that the material has a completely different morphology, where it appears as a long and well-structured nano-microfiber, with outer diameters of approximately $445 \pm 130 \mathrm{~nm}$. Adding the antigens in the hydrogel, Figures $3 \mathrm{~g}$ and $3 \mathrm{~h}$, the nano-microfibers presented the average diameter of $500 \pm 28 \mathrm{~nm}$ and the antigens are clearly observed on the structure and appear as an agglomerate of materials above the nano-microfibers; thus, the presence of the antigen in the matrix is confirmed.

Comparing both materials, when the hydrogel is structured in water, it appears with the usual fibrillated transparent hydrogel structure; in addition, in this case, the hydrogel is highly porous and can host different chemical species, such as the antigens of the studied case. However, when the hydrogel is structured in phosphate buffer solution, a transition from the transparent gel to the turbid gel occurs, which can be associated to morphological rearrangement of the fibers from randomly orientated fibrous network to large microstructures. This result corroborates with the rheology experiments, which will be discussed below, where the large storage modulus ( $\left.G^{\prime}\right)$ values measured for the hydrogel structured in water is higher as compared with the hydrogel prepared in PBS. The use of phosphate buffer to self-assemble the peptide hydrogel is due to the expected application of the buffer in

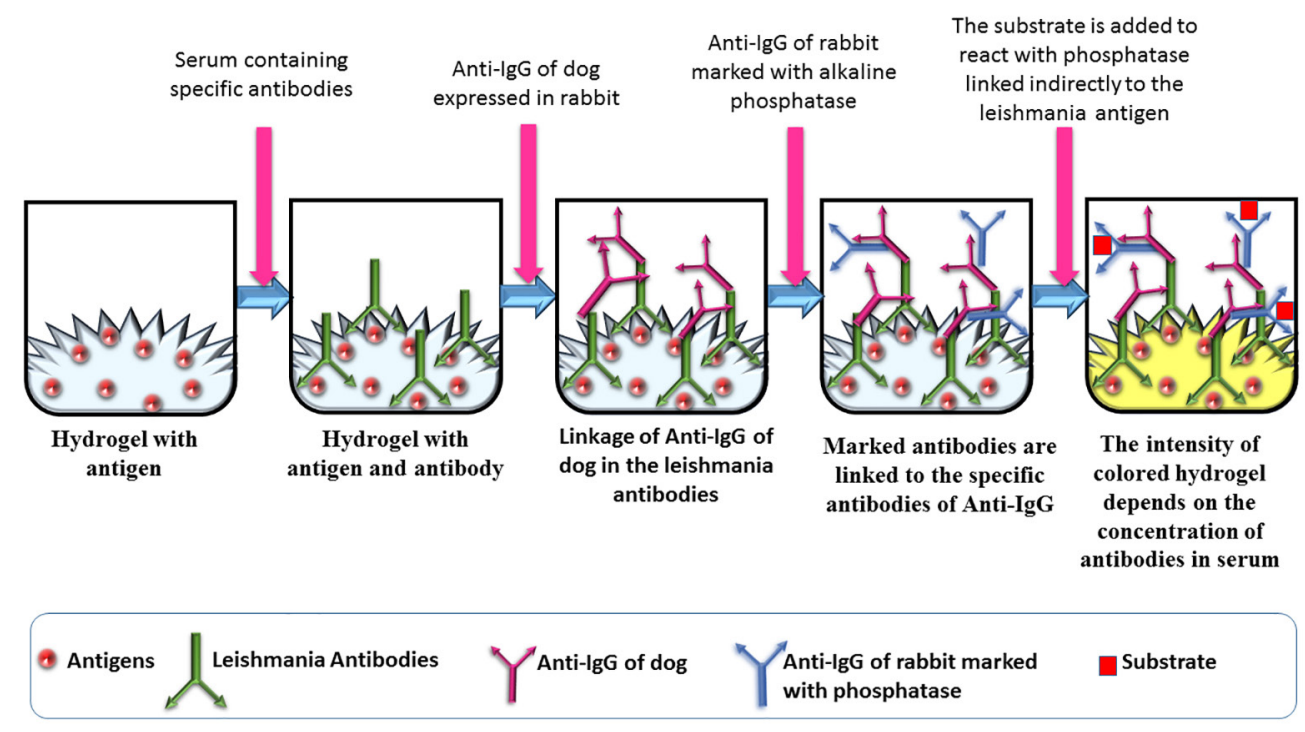

Figure 2. Schematic illustration of the enzyme-linked immunosorbent assay (ELISA) used with hydrogel samples. 

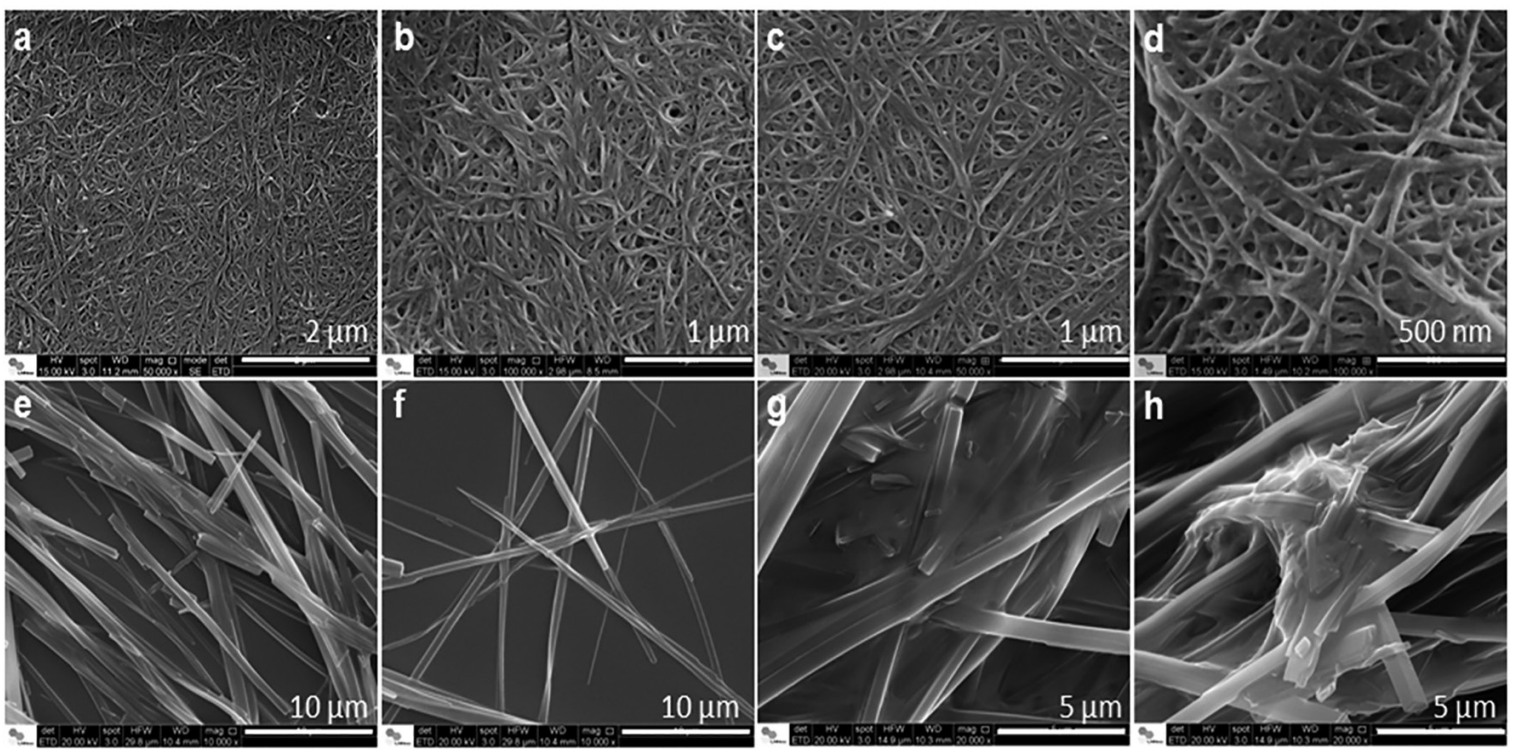

Figure 3. All the images were obtained using the SEM - Quanta-FEG-650. Images (a) and (b) refer to the hydrogel of Fmoc-FF structured in water, and images (c) and (d) are correlated to the same hydrogel in water structured with antigens. Images (e) and (f) are obtained with Fmoc-FF in PBS, and images (g) and (h) are correlated to the hydrogel structured in PBS containing antigens.

biological medium, such as in the canine serum attempted here and later with blood samples. Therefore, PBS has the property of acting as simulated body fluid, where PBS controls the $\mathrm{pH}$; moreover, changes in the $\mathrm{pH}$ could lead to the disassembly of the peptide structure. ${ }^{49}$

Recently, the kinetics and thermodynamic factors that lead the peptides to self-assembly in hydrogels or organized nano-microcrystals were discussed. ${ }^{50,51}$ Moreover, the presence of species that changes the interaction of the peptides with the solvent, such as, the ionic strength or the presence of salts, were found to induce the formation of one of the structures. Here, the following likely occurs: the buffer ionic strength induced the crystallization of the peptide in well-ordered fibers, suggesting that the transition observed in the gel must be due to a purely morphological rearrangement of the fibers and not a chemical change on the molecular scale, as shown in Figure 3. The infrared spectroscopy and X-ray diffraction (XRD) analysis of the turbid and transparent gels did not show change in packing on the molecular level (data not shown). These results are similar to the case of its non-protected counterpart, FF, which goes through a gel to crystal-gel transition, exchanging the solvent from toluene to ethanol due to lower solubility of FF in ethanol solutions. ${ }^{52}$ Another interesting result is that in the hydrogel, the antigen is distributed throughout the peptide matrix, which is the desired result, whereas when in gel-crystal, it becomes a second phase of the material, not mixed with the structure.

This fiber morphology obtained in hydrogels functions as a scaffold for any biomolecule; in the case of antigens, such a scaffold is required because it can anchor the antigens in the entire matrix, allowing them to remain in a moist medium. Another important variable that will influence the application of the hydrogels is their mechanical properties; thus, the rheological behaviors were studied, with the results shown in Figure 4.

The results of the rheology revealed that all the studied materials presented the viscoelastic behavior of hydrogel. Note that, in all samples, the elastic component prevailed over the viscous one, evidencing the elastic solid behavior of the samples. G' (storage modulus) represents the elastic response component, and G" indicates the viscous response component (loss modulus) of the materials. G' measures the deformation energy stored during the shear process of a test material (i.e., the stiffness of the material), and G' represents the energy dissipated during shear (i.e., the flow or liquid-like response of the material). ${ }^{53}$

The elastic behavior of hydrogels were found to be sensible to the hydrogel structure and the influence of the solutions used, water or PBS, because the highest values of G' were presented by samples from hydrogel in water (HO), indicating that when changing the medium, more entanglements and more interactions occurred. According to the literature, the enhanced hydrogen bonding into the self-assembled peptide strengthen the stability of hydrogels. ${ }^{54}$

The Table 1 presents the coefficients obtained with equation 1 adjusted for the G' results of each hydrogel. The values of $\mathrm{n}$ and $\mathrm{S}$ allow for a quantitative estimation of the hydrogel's strength, whereas for much higher $\mathrm{S}$ and G' with a lower $\omega$, a higher strength is exhibited by the hydrogel, indicative of the increase of entanglements resulting from the solvent used. Even visually, at the macroscopic scale, 

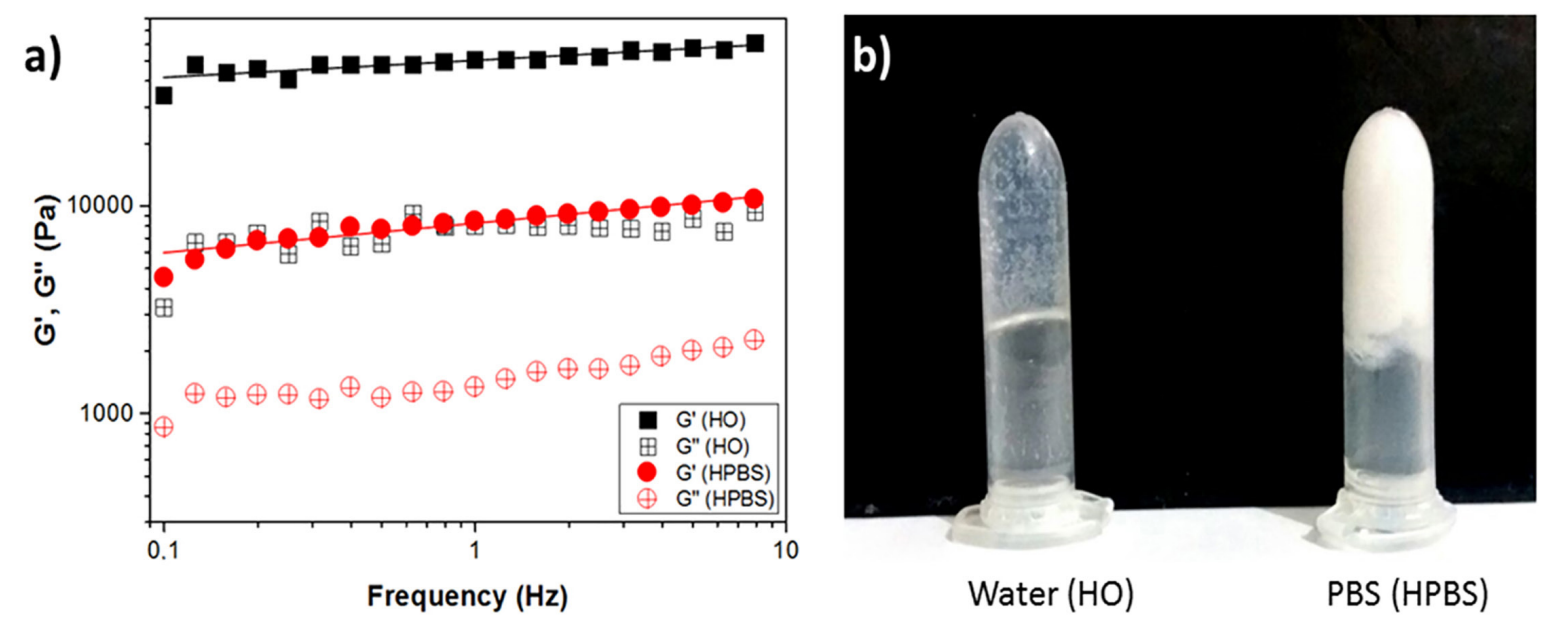

Figure 4. (a) Frequency sweep profile of the storage modulus G" (closed symbols) and the loss modulus G' (opened symbols) of hydrogels composed with water (HO) and hydrogels composed of PBS (HPBS). SDs have been omitted for clarity; however, in all cases, the coefficient of variation of triplicate analysis was less than 10\%; (b) inversion test performed with hydrogels of Fmoc-FF in water (HO) and in PBS (HPBS).

it was possible to note different features in both structures because two phases are observed when the peptide was structured with PBS, whereas when structured with water, it was in a homogenous phase.

Table 1. Coefficient of determination for linear regression $\left(R^{2}\right)$, storage modulus $\left(\mathrm{G}^{\prime}\right)$, gel strength $(\mathrm{S})$ and viscoelastic exponent $(\mathrm{n})$ of the hydrogels obtained

\begin{tabular}{lcccc}
\hline Hydrogel & $\mathrm{R}^{2}$ & $\mathrm{G}^{\prime} / \mathrm{Pa}$ & $\mathrm{S} / \mathrm{Pa}$ & $\mathrm{n}$ \\
\hline $\mathrm{H}_{2} \mathrm{O}$ & 0.82 & 50625 & $49934 \pm 600$ & $0.082 \pm 0.01$ \\
PBS & 0.93 & 8167 & $8171 \pm 101$ & $0.140 \pm 0.01$ \\
\hline
\end{tabular}

As known, hydrogels from Fmoc-FF are being studied for application in differentiation of stem cells $\mathrm{s}^{55-58}$ as well as in the proliferation of chondrocytes; ${ }^{21}$ in this sense, the matrix stiffness is quite important. ${ }^{59,60}$ In this direction, as discussed before, the hydrogel structure with water had higher mechanical strength; as a result, it was decided to study the hydrogel using water for the other experiments and mainly for the ELISA detection that uses canine serum because there are some difficulties related to obtaining this biological material extracted from live dogs.

The inner structure of our hydrogel matrices was probed using small-angle X-ray scattering (SAXS). SAXS is a powerful tool for in situ investigations at the nanoscale that enables unveiling of the structure of antigen-containing architectures under native conditions. In contrast to ex situ microscopy methods, which require drying procedure for preparing the samples, SAXS provides structural information in a hydrated environment, thus preserving the conformational states of the peptide/antigen matrices. We first assessed the behavior of antigens in aqueous solutions without the presence of Fmoc-FF in the medium. The main goal of these assays was to determine the average shape and sizes of the antigen on its own.

Small-angle X-rays scattering (SAXS) was performed to scrutinize the inner structure of the hydrogel matrices formulated above using water as a solvent. SAXS is a powerful tool for in situ investigations at the nanoscale that enables unveiling the structure of hydrogel-based architectures under native conditions. ${ }^{61-64}$ In contrast to $e x$ situ microscopy methods, which require drying procedures for preparing the samples, SAXS provides structural information within a hydrated environment, preserving the conformation of the peptide networks. ${ }^{64}$ The $q$-range accessed in our experiments corresponds to direct-space lengths ranging from ca. $2 \mathrm{~nm}$ to ca. $50 \mathrm{~nm}$; therefore, the SAXS data obtained here are related to the internal structure of the network revealed by SEM imaging (e.g., Figure 3d). Figure 5a shows SAXS plots from samples formulated with either pristine Fmoc-FF hydrogels or containing Leishmania infantum chagasi antigens at concentrations of 0.38 and $0.55 \mu \mathrm{g} \mathrm{mL}^{-1}$. The SASFit program ${ }^{65}$ was used to fit the data and extract quantitative information. The scattered intensities were fitted according to a singlecomponent form factor corresponding to the Porod's approximation for long cylinders, ${ }^{46}$ this approximation has been previously used for describing enzyme-derived hydrogels. ${ }^{66} \mathrm{~A}$ Gaussian contribution has been convoluted to the model to account for radii polydispersity, as described elsewhere..$^{18}$ In this way, the structural parameters arising from the modeling were the average radius $\mathrm{R}$ of cylinders and the corresponding polydispersity, $\Delta \mathrm{R}$, represented here by the standard deviation of the Gaussian distribution. The best fitting values emerging from this procedure reveal that the internal structure of the hydrogel networks is composed of highly polydisperse fibrillar sub-units with radii of only 
a few nanometers. The average radius arising from our fits performed in Fmoc-FF hydrogels was $\mathrm{R}=3.2 \pm 2.7 \mathrm{~nm}$. For antigen-containing formulations, the average radii were found at $\mathrm{R}=3.6 \pm 2.3 \mathrm{~nm}$ and $\mathrm{R}=4.0 \pm 2.9 \mathrm{~nm}$ for antigen concentrations of 0.38 and $0.55 \mu \mathrm{g} \mathrm{mL} \mathrm{m}^{-1}$, respectively. These values reveal that the sub-units of hydrogel networks are likely not affected upon the inclusion of antigens in the scaffold, representing an advantage because the main properties regarding structure and stability are preserved.

To obtain a greater direct-space visualization of the hydrogel networks, we performed transmission electron microscopy assays. In Figure 5b, a typical image is displayed together with magnifications from selected zones, where it is possible to observe the presence of fibrillar sub-units. The diameters of these structures are found to be between ca. $7 \mathrm{~nm}$ and ca. $14 \mathrm{~nm}$, consistent with the

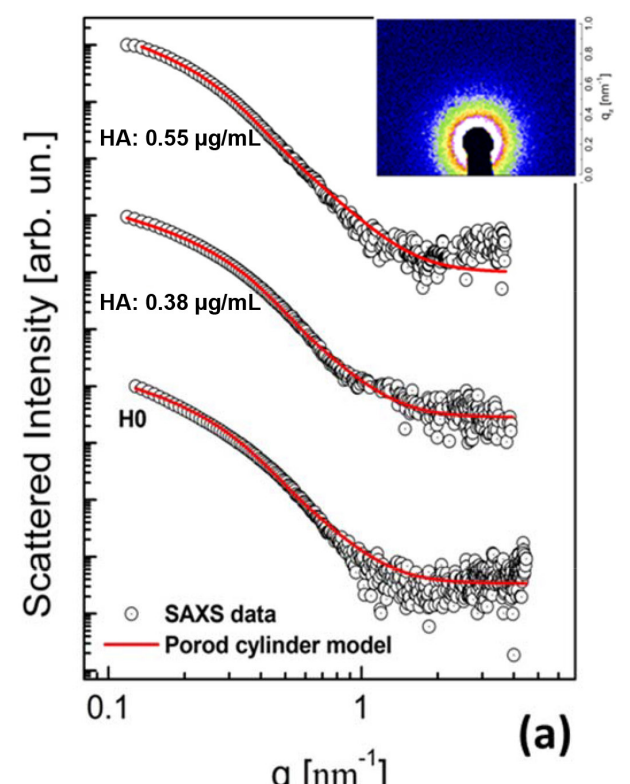

SAXS information derived above. In addition, coalescence between neighbor units during sample preparation for microscopy assays likely contributes to the presence of larger fibers observed in TEM images.

The sub-nanometer structure of the hydrogel matrices was probed using wide-angle X-ray scattering. Figure $5 \mathrm{c}$ shows a typical pattern arising from these assays, revealing the presence of a diffuse ring at q ca. $20 \mathrm{~nm}^{-1}$ accompanied by a shallow shoulder at q ca. $28 \mathrm{~nm}^{-1}$. These bands are related to the intermolecular correlations of water and are ascribed to a nearest-neighbor separation of d ca. $0.31 \mathrm{~nm}$ separation between oxygens in H-bonded water molecules, in close agreement with the characteristic patterns of bulk water. ${ }^{67}$ The presence of antigens in the hydrogel does not introduce noticeable changes at wide-angle region (data not shown). These findings not only confirm that Fmoc-FF
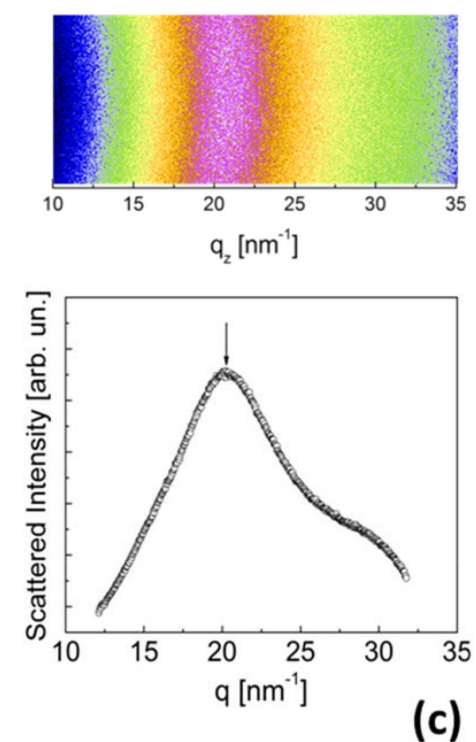

(c)

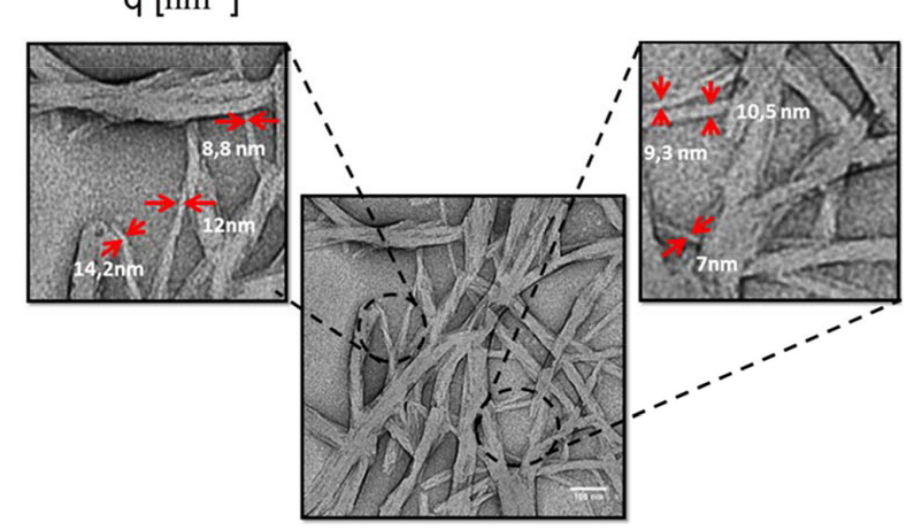

(b)

Figure 5. Scattering and microscopy data related to the local structure of Fmoc-FF hydrogels. (a) SAXS data from antigen-free and antigen-containing formulations. Red lines correspond to the model fitting used to extract quantitative information from the data (see text for details). Inset: 2D small-angle pattern from a hydrogel sample showing strong scattering at low-q region (data up to q ca. $1 \mathrm{~nm}^{-1}$ ); (b) TEM images from the antigen-free hydrogel (HO) showing sub-units made up from intertwined fibrils (red arrows in magnified pictures); (c) top: 2D WAXS pattern from a hydrogel matrix showing characteristic scattering from $\mathrm{H}$-bonded nearest-neighbor $\mathrm{H}_{2} \mathrm{O}$ molecules (bottom). 
matrices are mostly composed of water but also suggest large extents of fluid forming continuous zones in the interstice of the scaffold maintaining the same behavior of bulk water. This characteristic appears to be attractive because biomolecules dispersed into the liquid phase are prone to retain the properties exhibited in the bulk, that is, without interference from the host phase.

The efficient encapsulation of antigens by the hydrogels is a required feature to design a responsive hydrogel. The evaluation of the reaction between Leishmania antigens and antibodies was performed using the indirect enzymelinked immunosorbent assay (ELISA), which is extensively used in diagnostic laboratories or clinical testing. ${ }^{68}$ This technique is the preferred method to determine purified antibodies and can also be successfully employed for the qualitative and quantitative assessment of an antigen in a sample.$^{68,69}$ After the completion of the procedures detailed in the methods section, the results are shown in Figure 6.

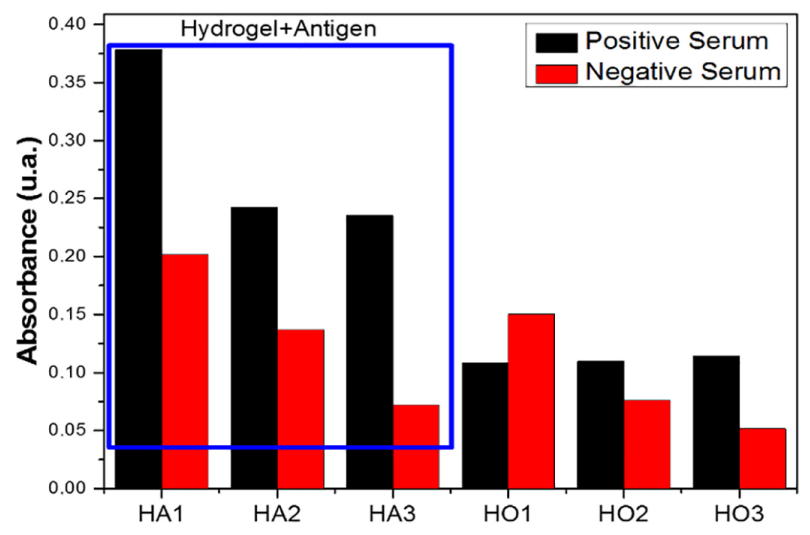

Figure 6. Results from the absorbance read after the ELISA experiment with positive and negative sera.

From the Figure 6, hydrogels containing Leishmania antigens (HA) presented great interaction with positive serum, as represented by the higher absorbance of it. This behavior occurs because of the recognition of the antigens presented in the hydrogel by the antibodies in positive serum, which was capable of permeating in the hydrogel structure. The hydrogel in contact with the higher concentration of serum $(1: 1 \mathrm{v} / \mathrm{v})$ exhibited a higher absorbance value of ca. 0.38 a.u. (HA1), compared to the hydrogel without antigen (HO) in contact with the positive serum at ca. 0.11 a.u., representing a nearly 4 -fold enhancement in the signal, showing that the hydrogel is able to detect the Leishmania antibodies in serum. For the other two dilutions of the serum in the hydrogel $(1: 10$ and $1: 40 \mathrm{v} / \mathrm{v})$, the absorbance values are approximately 0.24 a.u., whereas for the hydrogel without antigens, the value was approximately 0.10 a.u., showing that even in very diluted samples, it can show the presence of the leishmanial antibodies.
Therefore, the hydrogels containing antigens (HA1, HA2, and HA3) were found to exhibit some absorbance, even when reacted with the negative serum, with the absorbance intensity proportional to the serum concentration $(0.20$, 0.14 , and 0.08 a.u., respectively). These results indicate that there are some interactions between the compounds in blood fluid with the hydrogel containing antigens, probably soluble proteins or others antibodies, via intermolecular interactions. Because the hydrogel and the antigens are rich in functional groups that can accept or donate hydrogen bonds, it can easily adsorb compounds from the serum. This finding indicates that it is possible that the hydrogels without antigens (HO) also exhibit absorbance for the negative serum directly proportional to their concentration $(0.15$; 0.08 ; 0.05), showing that the hydrogel adsorbs compounds from that serum.

Moreover, it was shown that hydrogels can encapsulate the antigens and that they are sensitive to the presence of contaminated serum; however, more studies should be performed because there is no apparent selectivity with the antigen extraction procedure employed. It is known from the literature that it is difficult to separate the specific Leishmania genes; this difficulty is the motivation for many research studies of disease control, development of vaccines and others. ${ }^{69-71}$

The incorporation of biomolecules in the hydrogel structures can expand their properties and consequently favor new applications that require an interface with biological systems..$^{72}$ The moist environment present in all hydrogel material provides the adequate conditions to store biomolecules for long periods, thereby avoiding their degradations or denaturation. For example, enzymes, cells, some proteins and other biological entities require such environmental features. In this proof-of-concept work, it was shown that peptide hydrogel architecture provides a rational design for responsive peptide-based hydrogels. ${ }^{72,73}$ Some biosensors built with hydrogel systems have been studied, for example, the biochips for somatic mutation in genes detection, ${ }^{74}$ microfluidic devices sensitive to metalloproteinases that regulates the composition of an extracellular matrix and are correlated to some vascular disease, cancer progression, and bone disorders. ${ }^{41}$ In this sense, the opportunity of using hydrogels for antigens encapsulation can motivate the design of novel devices for disease diagnosis.

\section{Conclusions}

The system proposed of hydrogel based on Fmoc-FF was found to be adequate for the encapsulation of Leishmania antigens acting as scaffold for the soluble 
anchor. This hydrogel structure has the appropriate stiffness, as demonstrated with the rheology and microscopy results, and the SAXS results showed that the antigens did not modify the original structure of the nanofibers considerably. Furthermore, these materials are based on inexpensive starting materials, providing high responsiveness because they were able to detect the presence of antibodies in the positive serum. Although more studies should be performed in this area, this work represents a breakthrough in this application because, for the first time, the matrix of Fmoc-FF has been explored for use in the encapsulation of soluble proteins for the detection of negligible disease. In this case, we noted that this projected system using hydrogel allowed the stabilization of the antigens, thereby preserving their original features because of the moist environment provided by the hydrogel that was prepared in an aqueous solution. The opportunity of encapsulating antigens in peptide hydrogels can open up the appearance of new biosensing devices because they can store and preserve biomolecules for longer periods, thereby favoring the development of an instrument that is faster and easier to handle in the near future.

\section{Acknowledgments}

This work was supported by FAPESP (grant No. 2015/24018-1) and CNPq (grant No. 302923/2015-2, 400239/2014-0). INCT in Bioanalytics (FAPESP grant No. 08/57805-2 and CNPq grant No. 573672/2008-3) is kindly acknowledged for the grants. S. K. Jr. and E. R. S. acknowledge FAPESP for fellowships (Proc. No. 2012/01933-8 and 2013/12674-6). The Staff at LNNano and LNLS are kindly recognized for their invaluable help and providing access to SEM, TEM andSAXS beamtime (proposals SEM - 19955, TEM 19912 and SAXS1 20150184). Professor Cristiano Luis Pinto de Oliveira is kindly acknowledged for providing access to XENOCSXeuss machine for SAXS experiments at the University of São Paulo. Juliana dos Santos de Souza is acknowledged for acquiring TEM image. We are grateful to Dr Arlei Marcili from USP for providing the positive and negative sera for Leishmaniasis disease.

\section{References}

1. Feyzizarnagh, H.; Yoon, D.-Y.; Goltz, M.; Kim, D.-S.; Wiley Interdiscip. Rev.: Nanomed. Nanobiotechnol. 2016, 8, 730.

2. Adler-Abramovich, L.; Gazit, E.; Chem. Soc. Rev. 2014, 43, 6881.

3. Wang, Z.-G.; Ding, B.; Adv. Mater. 2013, 25, 3905.

4. Redaelli, F.; Sorbona, M.; Rossi, F. In Bioresorbable Polymers for Biomedical Applications; Perale, G.; Hilborn, J., eds.; Woodhead Publishing: Duxford, UK, 2017, p. 205.

5. Lian, M.; Chen, X.; Lu, Y.; Yang, W.; ACS Appl. Mater. Interfaces 2016, 8, 25036.

6. de la Rica, R.; Matsui, H.; Chem. Soc. Rev. 2010, 39, 3499.

7. Hamley, I. W.; Cheng, G.; Castelletto, V.; Macromol. Biosci. 2011, 11, 1068.

8. Parthasarathy, R. V.; Martin, C. R.; Nature 1994, 369, 298.

9. Yang, Y.; Khoe, U.; Wang, X.; Horii, A.; Yokoi, H.; Zhang, S.; Nano Today 2009, 4, 193.

10. Wang, Y. Q.; Zhang, Z. L.; Xu, L.; Li, X. Y.; Chen, H.; Colloid Surf., B 2013, 104, 163.

11. Mason, T. O.; Chirgadze, D. Y.; Levin, A.; Adler-Abramovich, L.; Gazit, E.; Knowles, T. P. J.; Buell, A. K.; ACS Nano 2014, 8, 1243.

12. Nelson, D. L.; Lehninger, A. L.; Cox, M. M.; Lehninger Principles of Biochemistry, $8^{\text {th }}$ ed.; W. H. Freeman: New York, USA, 2008.

13. Hartgerink, J. D.; Granja, J. R.; Milligan, R. A.; Ghadiri, M. R.; J. Am. Chem. Soc. 1996, 118, 43.

14. Voyer, N.; Top. Curr. Chem. 1996, 184, 1.

15. Azriel, R.; Gazit, E.; J. Biol. Chem. 2001, 276, 34156.

16. De Santis, E.; Ryadnov, M. G.; Chem. Soc. Rev. 2015, 44, 8288.

17. Dasgupta, A.; Mondal, J. H.; Das, D.; RSC Adv. 2013, 3, 9117.

18. Decandio, C. C.; Silva, E. R.; Hamley, I. W.; Castelletto, V.; Liberato, M. S.; Oliveira, V. X.; Oliveira, C. L. P.; Alves, W. A.; Langmuir 2015, 31, 4513.

19. Castelletto, V.; Gouveia, R. J.; Connon, C. J.; Hamley, I. W.; Seitsonen, J.; Ruokolainen, J.; Longo, E.; Siligardi, G.; Biomater. Sci. 2014, 2, 867.

20. Szkolar, L.; Guilbaud, J. B.; Miller, A. F.; Gough, J. E.; Saiani, A.; J. Pept. Sci. 2014, 20, 578.

21. Jayawarna, V.; Ali, M.; Jowitt, T. A.; Miller, A. F.; Saiani, A.; Gough, J. E.; Ulijn, R. V.; Adv. Mater. 2006, 18, 611.

22. Orbach, R.; Adler-Abramovich, L.; Zigerson, S.; MironiHarpaz, I.; Seliktar, D.; Gazit, E.; Biomacromolecules 2009, $10,2646$.

23. Seow, W. Y.; Hauser, C. A. E.; Mater. Today 2014, 17, 381.

24. Yan, X. H.; Zhu, P. L.; Li, J. B.; Chem. Soc. Rev. 2010, 39, 1877.

25. Fichman, G.; Gazit, E.; Acta Biomater. 2014, 10, 1671.

26. Liberato, M. S.; Kogikoski, S.; da Silva, E. R.; de Araujo, D. R.; Guha, S.; Alves, W. A.; J. Mater. Chem. B 2016, 4, 1405.

27. Souza, M. I.; Jaques, Y. M.; de Andrade, G. P.; Ribeiro, A. O.; da Silva, E. R.; Fileti, E. E.; Avilla Ede, S.; Pinheiro, M. V.; Krambrock, K.; Alves, W. A.; J. Phys. Chem. B 2013, 117, 2605.

28. Souza, M. I.; Silva, E. R.; Jaques, Y. M.; Ferreira, F. F.; Fileti, E. E.; Alves, W. A.; J. Pept. Sci. 2014, 20, 554.

29. Cipriano, T.; Knotts, G.; Laudari, A.; Bianchi, R. C.; Alves, W. A.; Guha, S.; ACS Appl. Mater. Interfaces 2014, 6, 21408.

30. Khanra, S.; Cipriano, T.; Lam, T.; White, T. A.; Fileti, E. E.; Alves, W. A.; Guha, S.; Adv. Mater. Interfaces 2015, 2, 1500265 . 
31. Ely, F.; Cipriano, T. C.; da Silva, M. O.; Peressinotto, V. S. T.; Alves, W. A.; RSC Adv. 2016, 6, 32171.

32. Bianchi, R. C.; da Silva, E. R.; Dall'Antonia, L. H.; Ferreira, F. F.; Alves, W. A.; Langmuir 2014, 30, 11464.

33. Sousa, C. P.; Coutinho-Neto, M. D.; Liberato, M. S.; Kubota, L. T.; Alves, W. A.; J. Phys. Chem. C 2015, 119, 1038.

34. Kogikoski, S.; Sousa, C. P.; Liberato, M. S.; Andrade-Filho, T.; Prieto, T.; Ferreira, F. F.; Rocha, A. R.; Guha, S.; Alves, W. A.; Phys. Chem. Chem. Phys. 2016, 18, 3223.

35. Cipriano, T. C.; Takahashi, P. M.; Lima, D.; Oliveira Jr., V. X.; Souza, J. A.; Martinho, H.; Alves, W. A.; J. Mater. Sci. 2010, 45, 5101.

36. Matos, I. O.; Alves, W. A.; ACS Appl. Mater. Interfaces 2011, $3,4437$.

37. Patrick, A. G.; Ulijn, R. V.; Macromol. Biosci. 2010, 10, 1184.

38. Thornton, P. D.; Mart, R. J.; Ulijn, R. V.; Adv. Mater. 2007, 19, 1252.

39. Kim, J. H.; Lim, S. Y.; Nam, D. H.; Ryu, J.; Ku, S. H.; Park, C. B.; Biosens. Bioelectron. 2011, 26, 1860.

40. Mathers, C. D.; Ezzati, M.; Lopez, A. D.; PLoS Neglected Trop. Dis. 2007, 1, e114.

41. Han, S. W.; Koh, W.-G.; Anal. Chem. 2016, 88, 6247.

42. Yemini, M.; Reches, M.; Gazit, E.; Rishpon, J.; Anal. Chem. 2005, 77, 5155 .

43. Tan, B.; Zhao, H.; Du, L.; Gan, X.; Quan, X.; Biosens. Bioelectron. 2016, 83, 267.

44. Smith, A. M.; Williams, R. J.; Tang, C.; Coppo, P.; Collins, R. F.; Turner, M. L.; Saiani, A.; Ulijn, R. V.; Adv. Mater. 2008, 20, 37.

45. Souto, D. E. P.; Silva, J. V.; Martins, H. R.; Reis, A. B.; Luz, R. C. S.; Kubota, L. T.; Damos, F. S.; Biosens. Bioelectron. 2013, 46, 22.

46. Bressler, I.; Kohlbrecher, J.; Thunemann, A. F.; J. Appl. Crystallog. 2015, 48, 1587.

47. Franke, D.; Svergun, D. I.; J. Appl. Crystallog. 2009, 42, 342.

48. Smith, A. M.; Williams, R. J.; Tang, C.; Coppo, P.; Collins, R. F.; Turner, M. L.; Saiani, A.; Ulijn, R. V.; Adv. Mater. 2008, 20, 37.

49. Nezammahalleh, H.; Amoabediny, G.; Kashanian, F.; Foroughi Moghaddam, M. H.; Results Phys. 2015, 5, 11.

50. Wang, J.; Liu, K.; Xing, R.; Yan, X.; Chem. Soc. Rev. 2016, 45, 5589.

51. Ramos Sasselli, I.; Halling, P. J.; Ulijn, R. V.; Tuttle, T.; ACS Nano 2016, 10, 2661.

52. Zhu, P.; Yan, X.; Su, Y.; Yang, Y.; Li, J.; Chem. - Eur. J. 2010 , $16,3176$.

53. Calixto, G.; Yoshii, A. C.; Rocha e Silva, H.; Cury, B. S. F.; Chorilli, M.; Pharm. Dev. Technol. 2015, 20, 490.

54. Gong, X.; Branford-White, C.; Tao, L.; Li, S.; Quan, J.; Nie, H.; Zhu, L.; Mater. Sci. Eng. C 2016, 58, 478.
55. Place, E. S.; George, J. H.; Williams, C. K.; Stevens, M. M.; Chem. Soc. Rev. 2009, 38, 1139.

56. Place, E. S.; Evans, N. D.; Stevens, M. M.; Nat. Mater. 2009 , $8,457$.

57. Lutolf, M. P.; Gilbert, P. M.; Blau, H. M.; Nature 2009, 462, 433.

58. Liu, S. Q.; Tay, R.; Khan, M.; Ee, P. L. R.; Hedrick, J. L.; Yang, Y. Y.; Soft Matter 2010, 6, 67.

59. Keung, A. J.; Kumar, S.; Schaffer, D. V. In Annual Review of Cell and Developmental Biology, vol. 26; Schekman, R.; Goldstein, L.; Lehmann, R., eds.; Annual Reviews: Palo Alto, USA, 2010, p. 533.

60. Engler, A. J.; Sen, S.; Sweeney, H. L.; Discher, D. E.; Cell 2006, 126, 677.

61. Krysmann, M. J.; Castelletto, V.; Kelarakis, A.; Hamley, I. W.; Hule, R. A.; Pochan, D. J.; Biochemistry 2008, 47, 4597.

62. Boothroyd, S.; Miller, A. F.; Saiani, A.; Faraday Discuss. 2013, 166, 195.

63. Tamon, H.; Ishizaka, H.; J. Colloid. Interface Sci. 1998, 206, 577.

64. Singh, A.; Suri, S.; Roy, K.; Biomaterials 2009, 30, 5187.

65. Pedersen, J. S.; Adv. Colloid Interface Sci. 1997, 70, 171.

66. De Leon-Rodriguez, L. M.; Kamalov, M.; Hemar, Y.; Mitra, A. K.; Castelletto, V.; Hermida-Merino, D.; Hamley, I. W.; Brimble, M. A.; Chem. Comm. 2016, 52, 4056.

67. Hura, G.; Sorenson, J. M.; Glaeser, R. M.; Head-Gordon, T.; J. Chem. Phys. 2000, 113, 9140.

68. Ferroglio, E.; Zanet, S.; Mignone, W.; Poggi, M.; Trisciuoglio, A.; Bianciardi, P.; Clin. Vaccine Immunol. 2013, 20, 657.

69. Suzuki, R. B.; Cabral, A. D.; Tonhosolo, R.; Marcili, A.; Sanches, C. O. C. C.; Martins, L. P. A.; Sperança, M. A.; J. Mol. Biomarkers Diagn. 2016, 7, 1000269.

70. Joshi, S.; Rawat, K.; Yadav, N. K.; Kumar, V.; Siddiqi, M. I.; Dube, A.; Front. Immunol. 2014, 5, 380.

71. Roatt, B. M.; Aguiar-Soares, R. D. O.; Vitoriano-Souza, J.; Coura-Vital, W.; Braga, S. L.; Corrêa-Oliveira, R.; MartinsFilho, O. A.; Teixeira-Carvalho, A.; de Lana, M.; Gontijo, N. F.; PLoS One 2012, 7, e49780.

72. Hamley, I. W.; Krysmann, M. J.; Castelletto, V.; Noirez, L.; Adv. Mater. 2008, 20, 4394.

73. Hamley, I. W.; Krysmann, M. J.; Castelletto, V.; Kelarakis, A.; Noirez, L.; Hule, R. A.; Pochan, D.; Chem. - Eur. J. 2008, 14, 11369.

74. Emelyanova, M.; Arkhipova, K.; Mazurenko, N.; Chudinov, A.; Demidova, I.; Zborovskaya, I.; Lyubchenko, L.; Zasedatelev, A.; Nasedkina, T.; Appl. Immunohistochem. Mol. Morphol. 2015, 23,255 .

Submitted: September 30, 2016 Published online: November 17, 2016 